\title{
The importance of nitrogen fixation to a temperate, intertidal embayment determined using a stable isotope mass balance approach
}

5 Douglas G. Russell ${ }^{1}$, Adam J. Kessler ${ }^{1}$, Wei Wen Wong ${ }^{1}$, and Perran L. M. Cook ${ }^{1}$

${ }^{1}$ Water Studies Centre, Monash University, Clayton, 3800, Australia.

Correspondence to: Douglas G. Russell (douglas.russell@monash.edu)

Contents of this file:

10

Figures $\mathrm{S} 1$ to $\mathrm{S} 3$

Table S1 to S2

Supplementary data:

15

Figure $\mathrm{S} 1$ provides the distribution of the model output for the sedimentary isotopic signature $\left(\delta^{15} \mathrm{~N}\right)$ described in our study.

The summary of results of the linear regression analysis for all parameters (loadings and isotopic end-members/fractionation factors) used in the model described in our study are presented in Figures S2-S3.

20 The coordinates for the oceanic and terrestrial field sites are summarised in Table S1. Table S2 summarises the output from the statistical analysis of the particulate nitrogen content and isotopic signature $\left(\delta^{15} \mathrm{~N}\right)$ over the tidal cycle at San Remo and Cowes. 


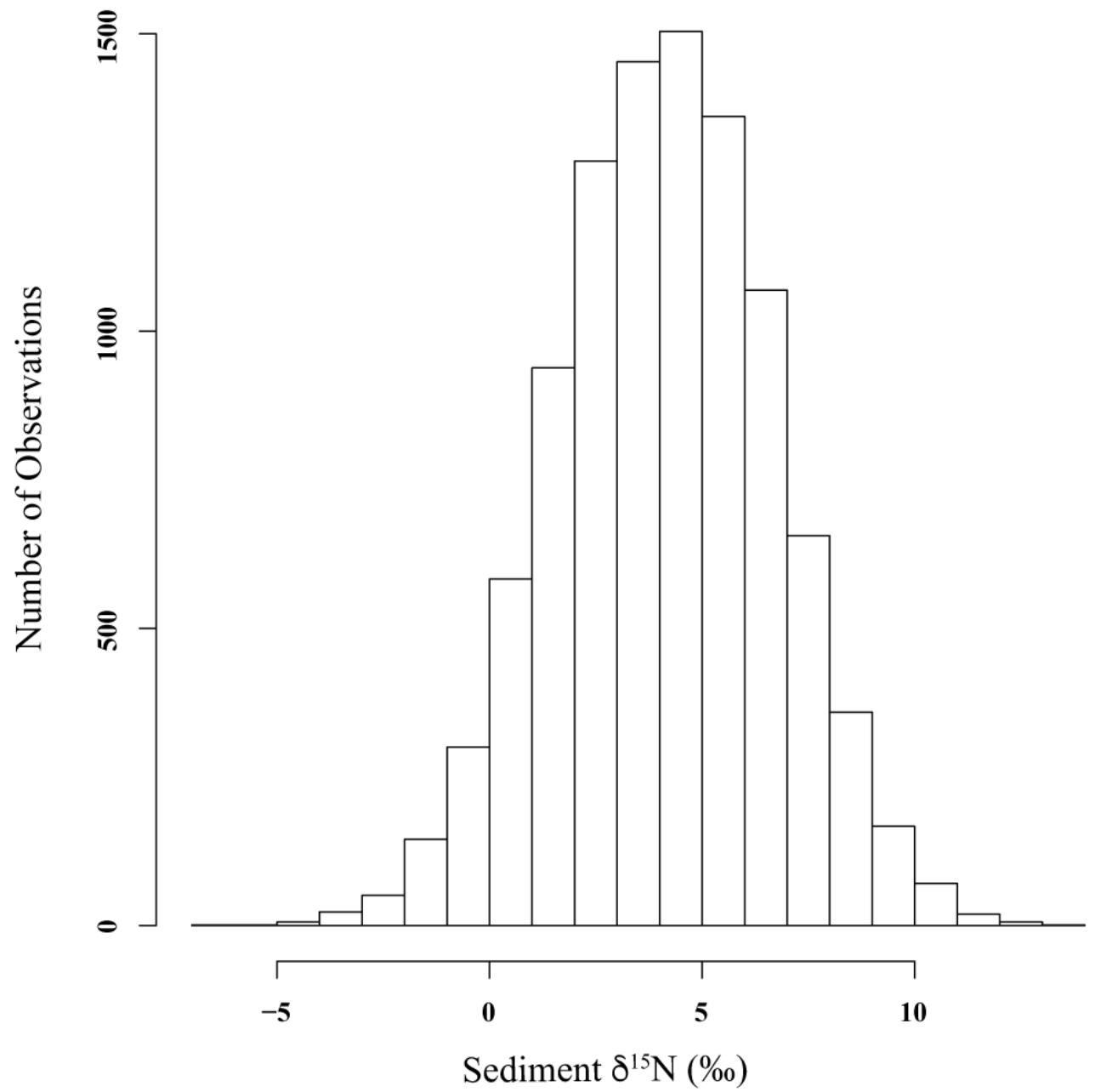

Figure S1: Distribution of model output after 10,000 iterations. 

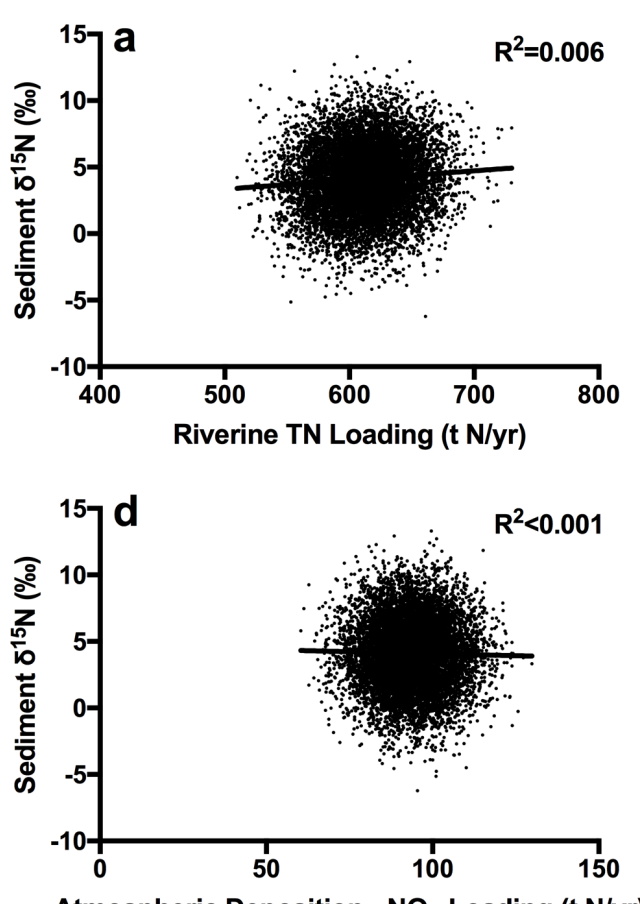

Atmospheric Deposition - $\mathrm{NO}_{\mathrm{x}}$ Loading (t N/yr)

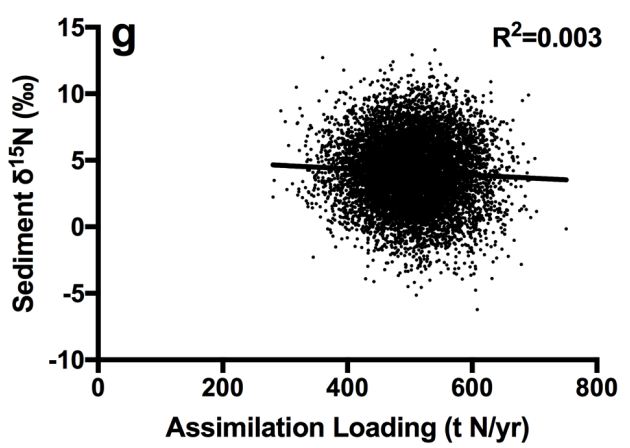

Figure S2: Linear regression analysis for each loading term vs. sediment $\delta^{15} \mathrm{~N}$. (a) Riverine TN, (b) nitrogen fixation, (c) atmospheric deposition - $\mathrm{NH}_{4}{ }^{+}$,

(d) atmospheric deposition - $\mathrm{NO}_{\mathrm{X}}$, (e) denitrification, (f) sediment burial and (g) assimilation.
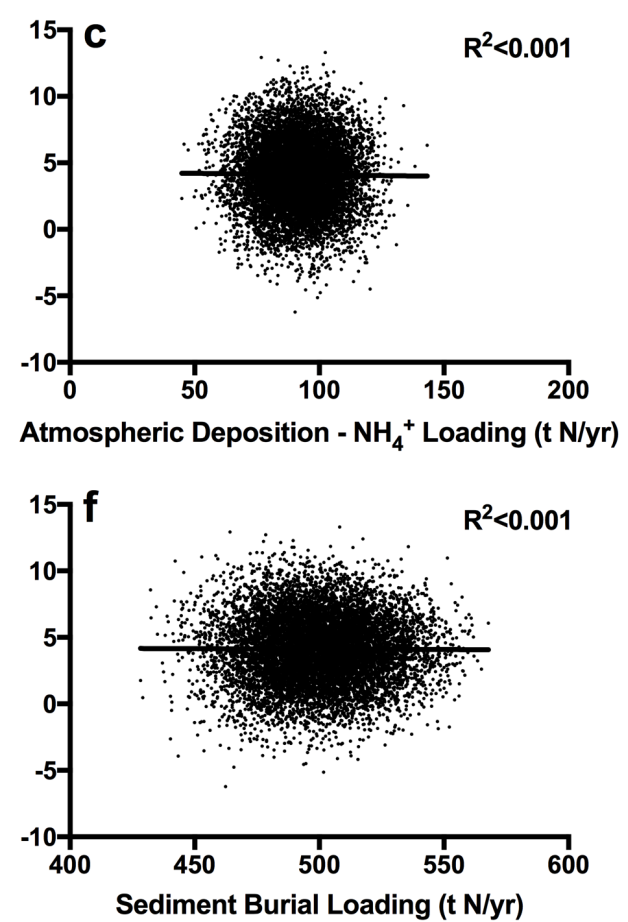

Sediment Burial Loading (t N/yr)
Denitrification Loading (t N/yr)
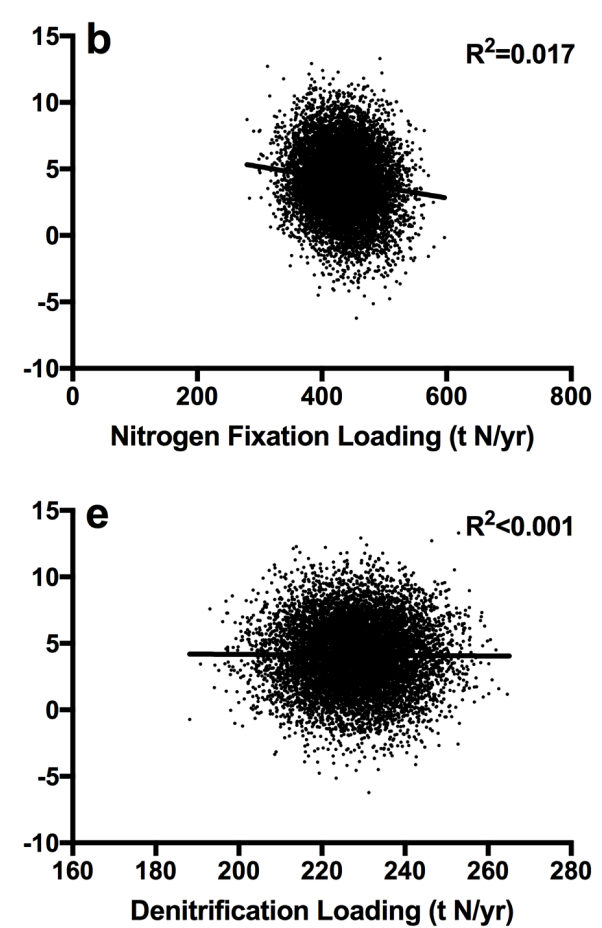

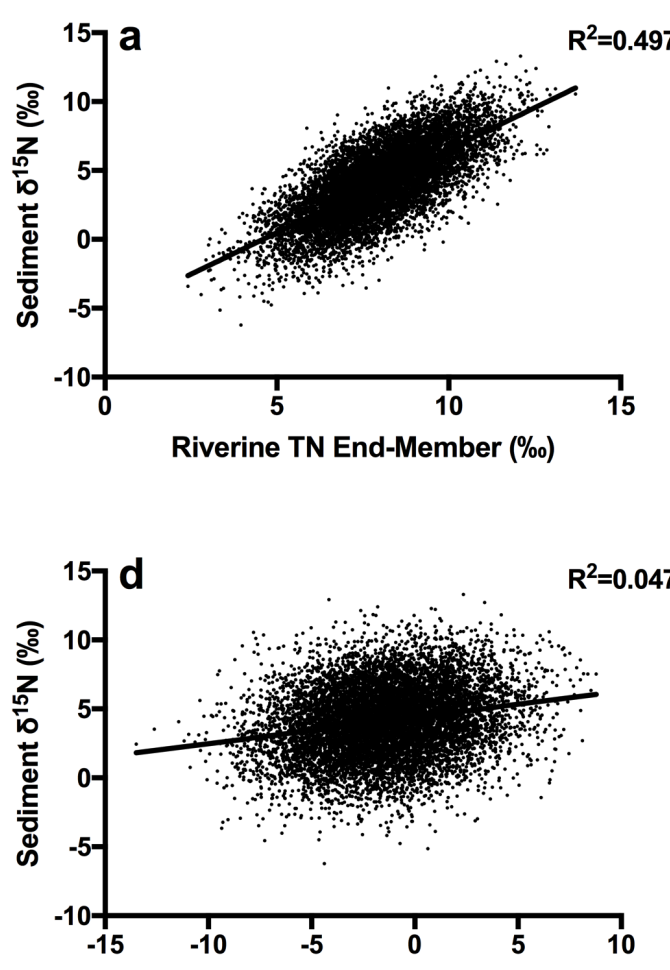

Atmospheric Deposition - $\mathrm{NO}_{\mathrm{X}}$ End-Member $(\%)$

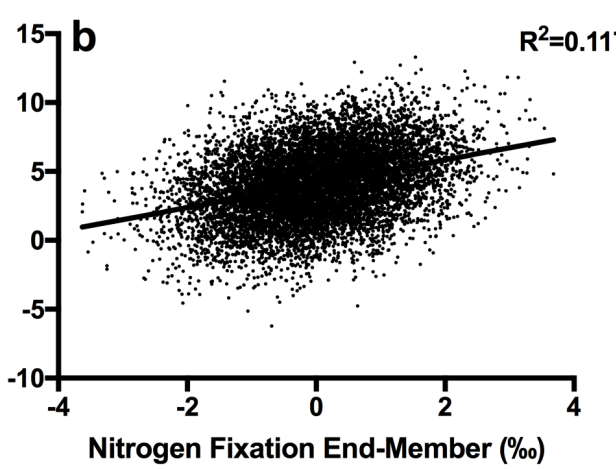

Nitrogen Fixation End-Member (\%o)

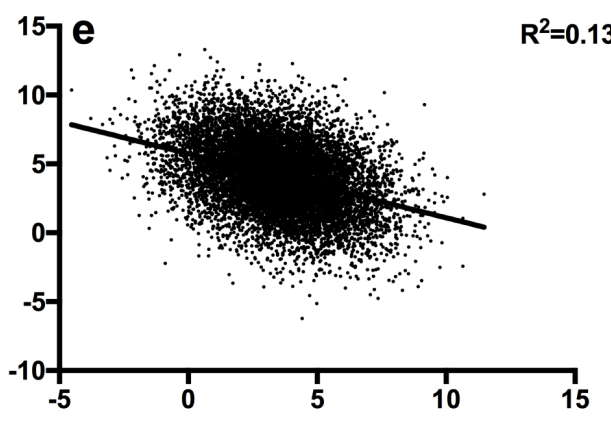

Denitrification Fraction Factor (\%)
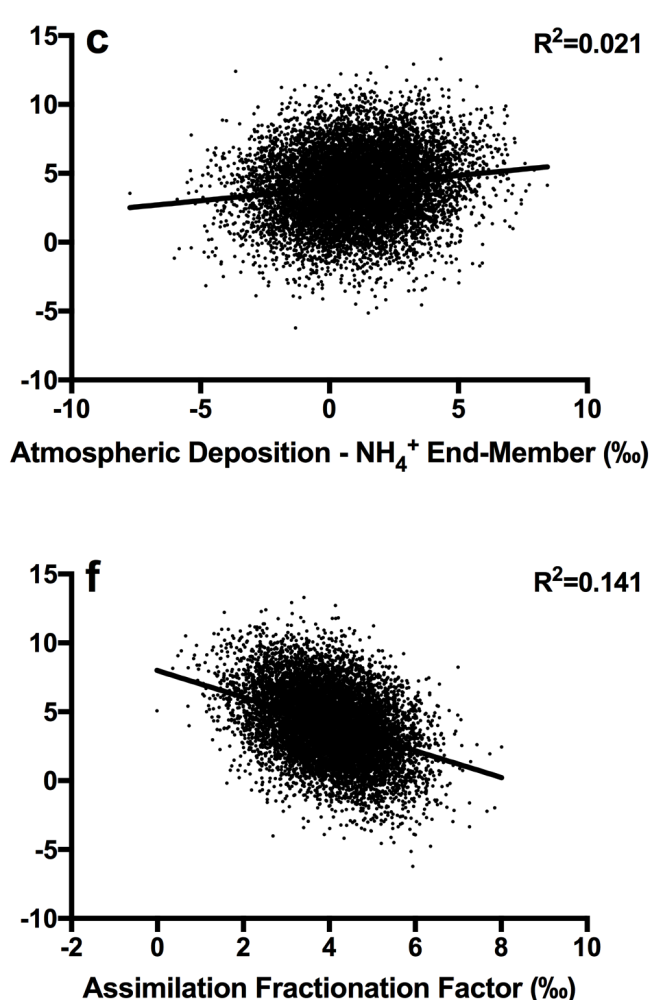

Figure S3: Linear regression analysis for each end-member vs. sediment $\delta^{15} \mathbf{N}$. (a) Riverine TN, (b) nitrogen fixation, (c) atmospheric deposition - $\mathrm{NH}_{4}{ }^{+}$, (d) atmospheric deposition - $\mathrm{NO}_{\mathrm{x}}$, (e) denitrification and (f) assimilation. 
Table S1: Sample locations for oceanic and riverine inputs.

\begin{tabular}{lll}
\hline Site & Coordinates & Terrestrial/Oceanic \\
\hline Bass River & $-38.467045^{\circ}, 145.515672^{\circ}$ & Terrestrial \\
Bunyip River & $-38.196927^{\circ}, 145.477834^{\circ}$ & Terrestrial \\
Lang Lang River & $-38.255695^{\circ}, 145.548879^{\circ}$ & Terrestrial \\
Toomuc Creek & $-38.178193^{\circ}, 145.441891^{\circ}$ & Terrestrial \\
Watsons Creek & $-38.229754^{\circ}, 145.235564^{\circ}$ & Terrestrial \\
Cowes & $-38.446132^{\circ}, 145.239837^{\circ}$ & Oceanic \\
San Remo & $-38.520262^{\circ}, 145.364816^{\circ}$ & Oceanic \\
\hline
\end{tabular}


Table S2: Results of two-factor ANOVA comparing the nitrogen isotopic signature $\left(\delta^{15} \mathrm{~N}\right)$ and nitrogen content of oceanic-derived particulate material amongst sites (Cowes and San Remo) and tidal cycle (incoming and outgoing tides). *Denotes $p<0.05$.

\section{$\delta^{15} \mathrm{~N}$ of particulate material}

\begin{tabular}{llll}
\hline Source & $\mathbf{d F}$ & $\mathbf{F}$ & $\mathbf{P}$ \\
\hline Sample Site & 1 & 2.421 & 0.127 \\
Tide & 1 & 1.792 & 0.188 \\
Sample Site $\times$ Tide & 1 & 0.057 & 0.812
\end{tabular}

Nitrogen content of particulate material

\begin{tabular}{llll}
\hline Source & $\mathbf{d F}$ & $\mathbf{F}$ & $\mathbf{P}$ \\
\hline Sample Site & 1 & 17.763 & $<\mathbf{0 . 0 0 1 *}$ \\
Tide & 1 & 0.073 & 0.788 \\
Sample Site $\times$ Tide & 1 & 0.851 & 0.362
\end{tabular}

